\title{
Spin-down evolution and radio disappearance of the magnetar PSR J1622-4950
}

DOI:

10.3847/1538-4357/aa73de

\section{Document Version}

Final published version

Link to publication record in Manchester Research Explorer

\section{Citation for published version (APA):}

Scholz, P., Camilo, F., Sarkissian, J., Reynolds, J. E., Levin, L., Bailes, M., Burgay, M., Johnston, S., Kramer, M., \& Possenti, A. (2017). Spin-down evolution and radio disappearance of the magnetar PSR J1622-4950. The Astrophysical Journal, 841(2). https://doi.org/10.3847/1538-4357/aa73de

\section{Published in:}

The Astrophysical Journal

\section{Citing this paper}

Please note that where the full-text provided on Manchester Research Explorer is the Author Accepted Manuscript or Proof version this may differ from the final Published version. If citing, it is advised that you check and use the publisher's definitive version.

\section{General rights}

Copyright and moral rights for the publications made accessible in the Research Explorer are retained by the authors and/or other copyright owners and it is a condition of accessing publications that users recognise and abide by the legal requirements associated with these rights.

\section{Takedown policy}

If you believe that this document breaches copyright please refer to the University of Manchester's Takedown Procedures [http://man.ac.uk/04Y6Bo] or contact uml.scholarlycommunications@manchester.ac.uk providing relevant details, so we can investigate your claim.

\section{OPEN ACCESS}




\title{
Spin-down Evolution and Radio Disappearance of the Magnetar PSR J1622-4950
}

\author{
P. Scholz ${ }^{1,2}$, F. Camilo ${ }^{3}$, J. Sarkissian ${ }^{4}$, J. E. Reynolds ${ }^{5}$, L. Levin ${ }^{6}$, M. Bailes ${ }^{7,8}$, M. Burgay ${ }^{9}$, \\ S. Johnston ${ }^{5}$, M. Kramer ${ }^{6,10}$, and A. Possenti ${ }^{9}$ \\ ${ }^{1}$ National Research Council of Canada, Herzberg Astronomy and Astrophysics, Dominion Radio Astrophysical Observatory, P. O. Box 248, \\ Penticton, BC V2A 6J9, Canada; paul.scholz@nrc-cnrc.gc.ca \\ ${ }^{2}$ Department of Physics and McGill Space Institute, Rutherford Physics Building, McGill University, 3600 University Street, Montreal, Quebec, H3A 2T8, Canada \\ ${ }^{3}$ SKA South Africa, Pinelands, 7405, South Africa \\ ${ }^{4}$ CSIRO Parkes Observatory, Parkes, NSW 2870, Australia \\ ${ }^{5}$ CSIRO Astronomy and Space Science, Australia Telescope National Facility, Epping, NSW 1710, Australia \\ ${ }^{6}$ Jodrell Bank Centre for Astrophysics, School of Physics and Astronomy, The University of Manchester, Manchester M13 9PL, UK \\ ${ }^{7}$ Centre for Astrophysics and Supercomputing, Swinburne University of Technology, Mail H30, P. O. Box 218, Hawthorn, VIC 3122, Australia \\ ${ }^{8}$ ARC Centre of Excellence for All-Sky Astronomy (CAASTRO), School of Physics, The University of Sydney, NSW 2006, Australia \\ 9 INAF-Osservatorio Astronomico di Cagliari, Via della Scienza 5, I-09047 Selargius (CA), Italy \\ ${ }^{10}$ Max-Planck-Institut für Radioastronomie, Auf dem Hügel 69, D-53121 Bonn, Germany \\ Received 2016 December 21; revised 2017 May 13; accepted 2017 May 15; published 2017 June 5
}

\begin{abstract}
We report on $2.4 \mathrm{yr}$ of radio timing measurements of the magnetar PSR J1622-4950 using the Parkes Observatory, between 2011 November and 2014 March. During this period the torque on the neutron star (inferred from the rotational frequency derivative) varied greatly, though much less erratically than during the 2 yr following its discovery in 2009. During the last year of our measurements the frequency derivative decreased in magnitude monotonically by $20 \%$, to a value of $-1.3 \times 10^{-13} \mathrm{~s}^{-2}$, a factor of 8 smaller than when it was discovered. The flux density continued to vary greatly during our monitoring through 2014 March, reaching a relatively steady low level after late 2012. The pulse profile varied secularly on a similar timescale as the flux density and torque. A relatively rapid transition in all three properties was evident in early 2013. After PSR J1622-4950 was detected in all of our 87 observations up to 2014 March, we did not detect the magnetar in our resumed monitoring starting in 2015 January and have not detected it in any of the 30 observations conducted through 2016 September.
\end{abstract}

Key words: pulsars: general - pulsars: individual (PSR J1622-4950) - stars: magnetars - stars: neutron

\section{Introduction}

Magnetars are a class of neutron stars with extremely high magnetic fields $\left(B \sim 10^{13-15} \mathrm{G}\right)$ and long spin periods $(2-12 \mathrm{~s})$. Their high-energy emission is powered via the decay of their magnetic fields, rather than through rotation. This is revealed through large outbursts and X-ray luminosities that exceed the available rotational spin-down luminosity (for reviews see Woods \& Thompson 2006; Mereghetti 2008). During outburst, magnetars can increase their X-ray fluxes by orders of magnitude and then fade on a timescale of months to years.

Most magnetars have been discovered and monitored in X-rays. The best characterized are those that have been monitored for more than 15 years with the Rossi X-Ray Timing Explorer (Dib \& Kaspi 2014), now continued by Swift (e.g., Archibald et al. 2013, 2015). This may be a biased sample, as only five of the 23 known magnetars (Olausen \& Kaspi 2014) ${ }^{11}$ are persistently bright enough to be monitored in this way (Dib \& Kaspi 2014). In order to expand our understanding of magnetars it is desirable to perform detailed, long-term monitoring of the rotational and radiative behavior of more objects.

Radio emission has been detected from only four of the known magnetars, but it is often quite bright (e.g., Camilo et al. 2007b; Shannon \& Johnston 2013). We can therefore expand the sample of well-characterized magnetars by performing long-term monitoring using radio telescopes. The study of radio emission from magnetars also provides a new

\footnotetext{
11 See the online Magnetar Catalog at http://www.physics.mcgill.ca/ pulsar/ magnetar/main.html.
}

electromagnetic window into the behavior of these most magnetic objects known.

XTE J1810-197 was the first magnetar to be detected in radio (Camilo et al. 2006), followed shortly thereafter by 1E 1547.0-5408 (Camilo et al. 2007b). They were found to have highly variable radio flux densities and pulse profiles, unlike ordinary pulsars. Very unusual compared to standard radio pulsars, their radio spectra are generally flat (e.g., Camilo et al. 2007c). Both are transient radio sources: radio emission from XTE J1810-197 turned off in 2008 (Camilo et al. 2016) and 1E 1547.0-5408 was detected intermittently following its 2009 outburst (Burgay et al. 2009; Camilo et al. 2009). The third radio magnetar, PSR J1622-4950, is the subject of this work. A fourth was more recently discovered $2^{\prime \prime}$ away from the Galactic center and is the closest known pulsar to Sgr A* (Eatough et al. 2013; Shannon \& Johnston 2013).

PSR J1622-4950 was discovered with the CSIRO Parkes telescope as a radio pulsar with a period of $P=4.3 \mathrm{~s}$ and a dispersion measure of DM $=820 \mathrm{pc} \mathrm{cm}^{-3}$ (Levin et al. 2010). To date it remains the only magnetar to have been detected in radio without prior knowledge of a corresponding X-ray source. Like other radio magnetars it has a flat spectrum, nearly $100 \%$ linear polarization, and highly variable flux density and pulse profiles. Its rotational behavior following discovery was characterized by Parkes observations between 2009 April and 2011 February (Levin et al. 2012). Long-term phase-connected timing solutions were not possible due to the rapidly evolving spin-frequency derivative, $\dot{\nu}$, and an insufficient observing cadence. From short-term timing solutions, $|\dot{\nu}|$ was found to have decreased by a factor of 2 in the $2 \mathrm{yr}$ following discovery. 
PSR J1622-4950 was identified as an X-ray source using archival and dedicated Chandra and XMM-Newton observations. Its X-ray flux decreased by a factor of $\sim 50$ between 2007 June and 2011 February, presumably following a prediscovery outburst. X-ray pulsations have not been detected, implying a 70\% limit on the pulsed fraction (Anderson et al. 2012).

Here we present the analysis and results of an additional $2.4 \mathrm{yr}$ of Parkes observations of PSR J1622-4950. We describe our data set in Section 2. In Sections 3.1 and 3.2 we show the pulse profile and flux density evolution of the source. In Section 3.3 we present a timing analysis and the resulting phase-connected timing solutions. We discuss our results in Section 4 and conclude in Section 5.

\section{Observations}

We observed PSR J1622-4950 with the Parkes telescope on a regular basis between 2011 November and 2014 March. These observations were typically conducted on the same days as those when we monitored the magnetar 1E 1547.0-5408, which was largely observed at frequencies near $3 \mathrm{GHz}$ because severe scattering renders its pulse hard to detect at $1.4 \mathrm{GHz}$ (Camilo et al. 2007b). We made a total of 87 observations on 81 days, $90 \%$ of them at $3 \mathrm{GHz}$ using the $10-50 \mathrm{~cm}$ receiver, and the remainder at $1.4 \mathrm{GHz}$ using the center beam of the $20 \mathrm{~cm}$ multibeam receiver (Staveley-Smith et al. 1996), once every 10 days on average. Integration times were typically 5 or 10 minutes per observation.

A total of 69 observations through 2013 September were conducted with the analog filterbank system (AFB; see, e.g., Manchester et al. 2001), used to sample a bandwidth of $864 \mathrm{MHz}$ centered on $3078 \mathrm{MHz}$ or a $288 \mathrm{MHz}$ band centered on $1374 \mathrm{MHz}$. In each case the individual channel widths were $3 \mathrm{MHz}$. In 2013 April we began using PDFB3, a digital filterbank (DFB), centered on $3100 \mathrm{MHz}$ to sample $5122 \mathrm{MHz}$-wide channels. In all cases we recorded totalintensity (polarization-summed) search-mode data using $1 \mathrm{~ms}$ samples.

Each of the data sets was subsequently dedispersed and folded using a known ephemeris (Levin et al. 2010). Each folded observation was inspected for frequency channels and subintegrations that were highly contaminated by radio frequency interference (RFI). The contaminated channels and subintegrations were then masked in all subsequent analysis.

PSR J1622-4950 was detected in every observation we made during 2011-2014. We resumed observations on 2015 January 11 but have not detected the pulsar in any of 30 epochs through 2016 September 16. These observations, largely at $3 \mathrm{GHz}$ using the PDFB4 DFB, lasted for 15 minutes on average.

In addition to the new observations conducted between 2011 and 2014, we use the flux densities and pulse times-of-arrival (TOAs) reported in Levin et al. (2012) from Parkes observations between 2009 and 2011. We also utilize 26 archival observations made between the data set presented in Levin et al. (2012) and the beginning of our campaign. These observations included 15 observations at $1.4 \mathrm{GHz}$ and three observations at $3.1 \mathrm{GHz}$ using PDFB3 $/ 4$, and eight $1.4 \mathrm{GHz}$ observations with the CASPER-Parkes-Swinburne Recorder.

\section{Analysis and Results}

\subsection{Pulse Profile Variations}

Similar to what was observed between 2009 and 2011 by Levin et al. (2012), the pulse profile of PSR J1622-4950 in our observations is made up of multiple components that vary in relative amplitude and separation over time. Figure 1 shows the profiles for all of our 2011-2014 observations. Long-period pulsars observed with the AFB system display artifacts caused by a high-pass filter with a $\approx 0.9 \mathrm{~s}$ time constant. We used the prescription given by Manchester et al. (2001) to correct for this effect in the profiles presented in Figure 1.

In late 2011, the profiles were clearly composed of two peaks, with the second fainter than the first. In late 2012, the pulsar became significantly fainter (see Section 3.2 ) and was more affected by RFI. Often the profile could only be resolved as a broad single peak. This persisted until late 2013, when the flux density increased slightly and the pulse profile narrowed (Figure 1).

To quantify the narrowing of the pulse profile, we fit a twoGaussian model to the profiles. The model fit to the profiles is

$$
\begin{aligned}
P\left(\phi, A_{i}, \mu_{i}, \sigma_{i}\right)= & A_{1} \exp \frac{-\left(\phi-\mu_{1}\right)^{2}}{2 \sigma_{1}^{2}} \\
& +A_{2} \exp \frac{-\left(\phi-\mu_{2}\right)^{2}}{2 \sigma_{2}^{2}},
\end{aligned}
$$

where $A_{i}$ are the amplitudes, $\mu_{i}$ are the peak phases, and $\sigma_{i}$ are the widths of the Gaussian components. The FWHM of the components is $2 \sqrt{2 \ln 2} \sigma_{i}$. The results of these fits are shown in Figure 2 and clear evolution is evident. The leading component remained relatively constant in width and the trailing Gaussian component became narrower and closer in phase to the first component as a function of time. This change was occurring on a similar timescale as the radio flux density decrease (see Section 3.2).

\subsection{Flux Density Evolution}

Our filterbank data were not flux calibrated. Nevertheless, we could extract useful flux density measurements by computing the area under each profile and scaling it to a Jansky scale using the system equivalent flux density (SEFD) at the location of the pulsar. First we set the off-pulse level to zero and normalized the summed pulse profile counts by the off-pulse rms. We then scaled the profile into units of flux density using the off-pulse rms from the radiometer equation (Dewey et al. 1985):

$$
\frac{\beta \text { SEFD }}{\sqrt{n_{p} t_{\text {int }} \Delta f}}
$$

where $\beta$ is a loss factor due to the digitization of the signal (1.5 for the AFB, 1.1 for the DFB), $n_{p}=2$ is the number of polarizations summed, $t_{\mathrm{int}}$ is the integration time per phase bin, and $\Delta f$ is the bandwidth. We determined the SEFD by analyzing with PSRCHIVE (Hotan et al. 2004) full-Stokes calibrated observations made with PDFB3. The SEFD at $3.1 \mathrm{GHz}$ was $61 \mathrm{Jy}$ (based on an observation done on 2011 December 12), while the SEFD at $1.4 \mathrm{GHz}$ was $69 \mathrm{Jy}$ (based on an observation done on 2010 November 3), both measured with about a $5 \%$ precision. Flux densities measured in this way are 

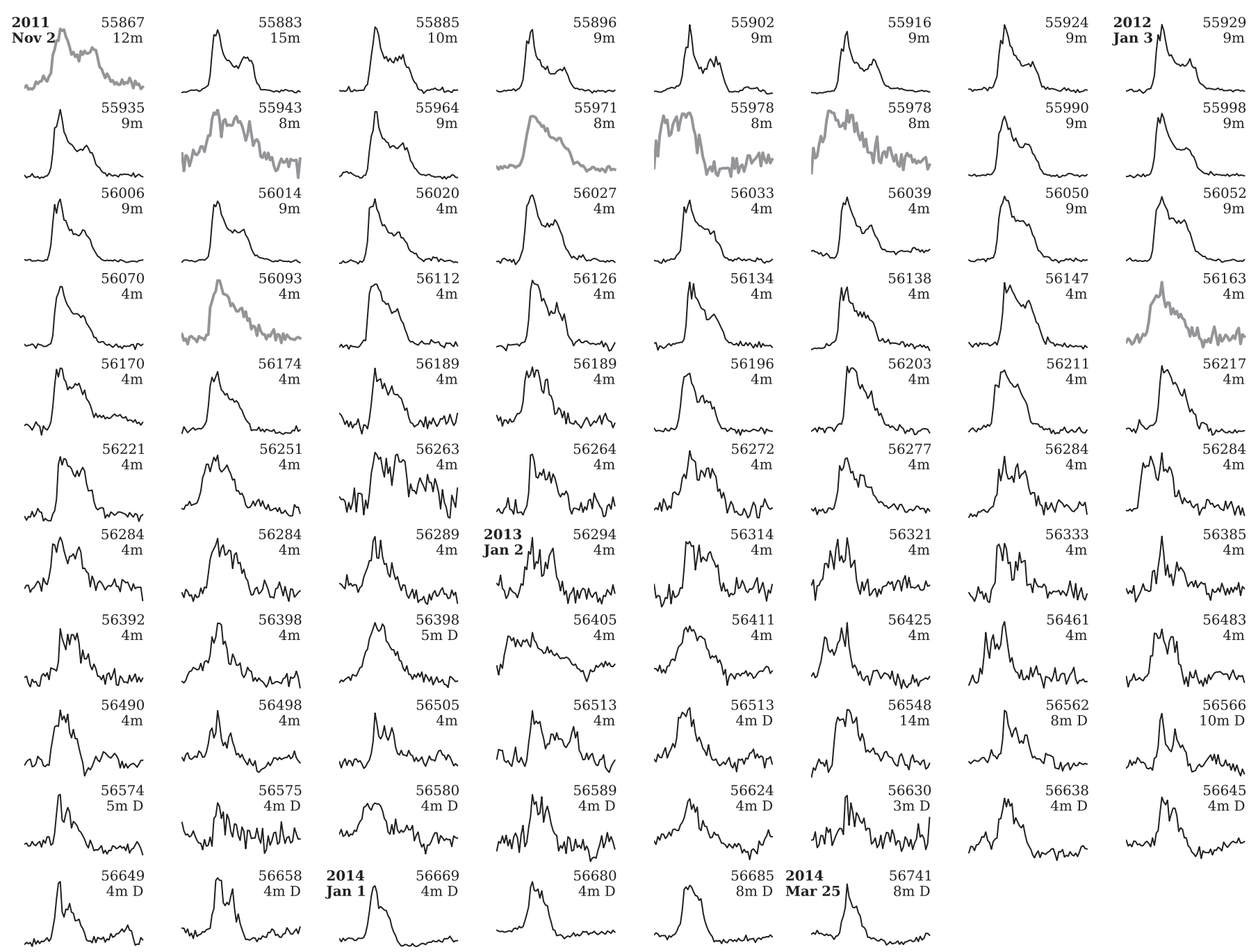

Figure 1. Radio pulse profiles of PSR J1622-4950. Black (gray) profiles correspond to $3 \mathrm{GHz}(1.4 \mathrm{GHz}$ ) observations. The full pulse period of $4.3 \mathrm{~s}$ is displayed, with 64 phase bins, and profiles are arbitrarily aligned. We list the MJD and integration time (in minutes) of each observation, along with calendar dates in select instances. Those observations that used a DFB are denoted by a "D." All other profiles, obtained with an AFB, have been corrected to account for instrumental artifacts (see Section 3.1). Some profiles remain somewhat contaminated by RFI.

shown in Figure 3. In the absence of residual RFI and other profile artifacts, we estimate that the absolute fractional uncertainty for each measurement was $\approx 25 \%$. However, some profiles were contaminated by residual RFI (see Figure 1). To address this, we made the measurements using two independent tools: one in which the off-pulse regions are chosen arbitrarily by a user and one in which the off-pulse regions are determined automatically by growing the off-pulse region until the variance of the off-pulse data changes by more than $10 \%$. These two tools yield different off-pulse baseline estimations, normalizations, and flux density values. While on occasion the two measures differed by up to $\approx 50 \%$, in most cases they agreed more closely and these discrepancies do not affect the trends visible in Figure 3.

Following the PSR J1622-4950 discovery, Levin et al. (2010) realized that prediscovery observations existed for the years 1998-2006 in the form of archival search-mode data for two nearby pulsars: PSR J1623-4949 (11' away) and PSR J1622-4944 ( $7^{\prime}$ away). When pointing at the latter, PSR J1622-4950 was near the half-power point of the Parkes $1.4 \mathrm{GHz}$ primary beam $(\mathrm{FWHM}=14 ! 4)$, with a reduction in sensitivity by a factor of 1.8 . When pointing at the former, the sensitivity was reduced by a factor of 6.3 assuming a Gaussian beam (which may not be appropriate so far off boresight). Astonishingly, Levin et al. (2010) recovered many bright detections of PSR J1622-4950 and estimated flux densities, even that far off axis. We have reanalyzed those data (52 individual AFB observations) in the same manner as for our new data set in order to place both sets of detections on the same flux density scale. We made 14 detections in prediscovery data, the same as Levin et al. (2010).

In Figure 3 we also include the flux densities corresponding to the data presented in Levin et al. (2010). However, Levin et al. (2010) used SEFD $=T_{\text {sys }} /$ Gain $=24 \mathrm{~K} / 0.735 \mathrm{~K} \mathrm{Jy}^{-1}=33 \mathrm{Jy}$, which is a factor of 2.1 less than our measured value of $69 \mathrm{Jy}$ at $1.4 \mathrm{GHz}$. In addition, they used a loss factor of $\beta=1.0$, while $\beta=1.1$ for the DFB data and $\beta=1.5$ for the AFB data. Thus we multiply the flux density values presented in Levin et al. (2010) by 2.3 for the DFB data (obtained during 2009-2011) and 3.1 for the AFB data (prior to 2007). Our SEFD was measured at the position of PSR J1622-4950, and the correction factor for the pre-2007 data (during which the telescope was pointed several arcminutes away from PSR J1622-4950) is therefore uncertain. Nevertheless, 


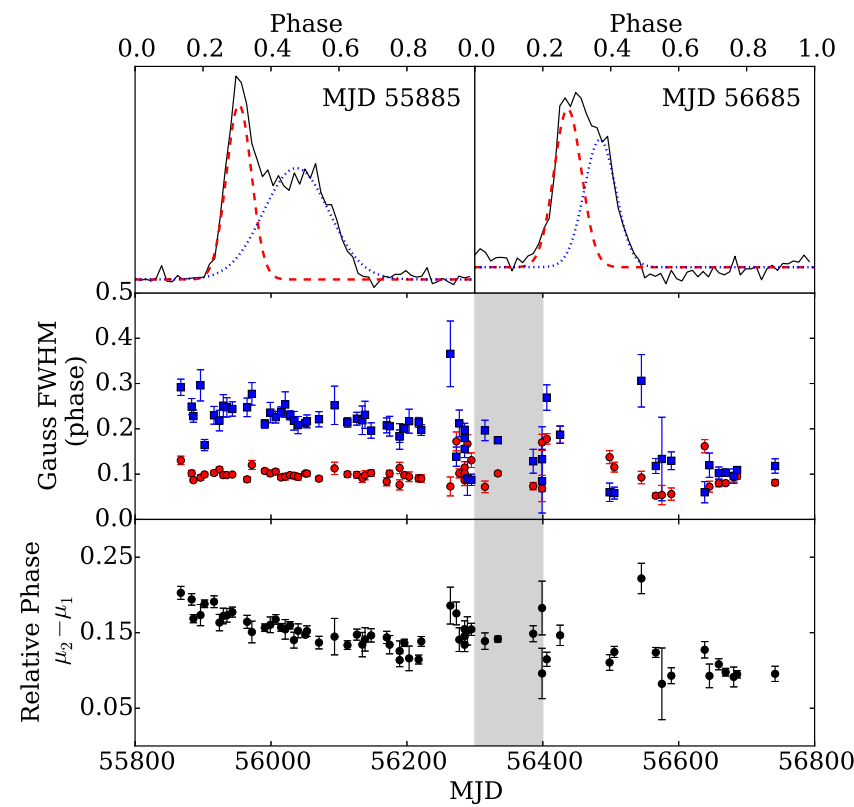

Figure 2. Gaussian fits to the pulse profiles of PSR J1622-4950. Top panel: two example profiles are shown with their two-component Gaussian fits shown. Middle panel: the FWHM of the two Gaussian components for each profile. Bottom panel: the separation between the peak phases of the two Gaussian components for each profile. The gray bar marks the period between MJD 56300 and MJD 56400 in order to illustrate the correlated behavior between the flux density (Figure 3), spin-down (Figure 4), and pulse profile evolution at that time.

we judge that our SEFD is a closer approximation to the true value than the cold-sky value assumed in Levin et al. (2010).

All flux density measurements are summarized in Figure 3. Many of the prediscovery values are much larger, as well as more variable, than the more recent ones. Another notable point is that we have numerous detections in 2012-2014 with flux densities below the (corrected) $3.8 \mathrm{mJy}$ detection limit of the off-axis observations (Levin et al. 2010 used a limit of $1.2 \mathrm{mJy}$ ). Therefore, it is quite possible that some nondetections for the 1998-2006 period (Levin et al. 2010) simply reflect a lack of sensitivity and that the pulsar would have been detected had it been observed on-axis. Those nondetections hence do not necessarily imply a turnoff in radio emission. By contrast, our consistent nondetections starting in 2015 (Section 2) reflect a different state compared to those noted in 2009-2014. For the first time in the study of PSR J1622-4950, we can entertain the possibility that the radio emission effectively turned off or at least transitioned to a significantly fainter state.

\subsection{Phase-coherent Timing}

In principle, the timing of radio magnetars presents particular challenges owing to the varying pulse profiles. In practice, for PSR J1622-4950 this did not present substantial difficulties for the post-2011 data used in this paper.

To account for coarse changes in pulse profiles, we used three separate templates for TOA extraction. For observations prior to MJD 56250, we used the profile observed on MJD 55924 as the template. For AFB observations from MJD 56250 onward, the MJD 56284 profile was used. Finally, the MJD 56685 profile was used to extract TOAs from all DFB observations (see Figure 1). All TOAs were obtained with the PRESTO (Ransom et al. 2002) tool get_TOAs.py.
In order to quantify the effect of the evolving profiles on the accuracy of the TOAs, we also extracted TOAs using templates built from multi-Gaussian fits to the profiles observed on MJD 55924 (for the AFB data) and MJD 56669 (for the DFB data). We then measured the difference between the corresponding original TOAs and the Gaussian-template TOAs. The standard deviation of these differences ranged over $20-40 \mathrm{~ms}$ for AFB TOAs and was $30 \mathrm{~ms}$ for the DFB TOAs, i.e., about $1 \%$ of the pulse period. We added these standard deviations in quadrature to our nominal TOA uncertainties to account for the error introduced in timing the pulsar with a restricted set of templates in the face of varying pulse profiles. One observation, on MJD 56545, was too faint to provide a reliable TOA.

The TOAs were fit to a timing model describing the pulsar rotation where the pulse phase as a function of time is described by a Taylor series expansion. Initially, only the spin frequency $\nu=1 / P$ was fit for, to a set of four TOAs extracted from each observation. A frequency derivative $\dot{\nu}$ was estimated from those measurements and was used as a starting point for the iterative process of long-term phase connection using TEMPO2 (Hobbs et al. 2006). For the final fits we extracted one TOA per observation in order to improve parameter precision.

Using simple timing models with only $\nu, \dot{\nu}$, and $\ddot{\nu}$, it is possible to phase-connect the data set in two separate date ranges. These solutions are shown in Table 1. In Figures 4(a) and (b), the $\dot{\nu}$ evolution and the phase residuals of these two solutions are shown in red and blue. In order to probe the evolution of $\dot{\nu}$ in more detail, we also fit short-term overlapping timing models using only $\nu$ and $\dot{\nu}$. Each short-term model was fit over a minimum of five observations spanning a minimum of 61 days and a maximum of 100 days. The resulting values of $\dot{\nu}$ are shown in Figure 4(a), where the horizontal bars represent the time span of the fits.

Formal pulsar TOA uncertainties obtained from crosscorrelating observed profiles with templates are often somewhat underestimated. It is therefore standard practice to increase the TOA errors by a scaling error factor (EFAC) that yields a reduced $\chi^{2} \equiv 1$, ensuring more realistic parameter uncertainties. We determined that for our data set $\mathrm{EFAC}=1.3$ by considering short-term timing solutions where the effects of timing noise were negligible.

To probe the timing evolution between the end of the data set from Levin et al. (2012) and the beginning of our 2011-2014 campaign, we also extracted TOAs from 26 archival observations (Section 2) using PSRCHIVE's pat utility. We fit timing solutions with $\nu$ and $\dot{\nu}$ to these data in two time spans where phase connection was possible. These frequency-derivative measurements are shown as red crosses in Figure 5.

\section{Discussion}

PSR J1622-4950 is the only magnetar whose rotation has been studied exclusively at radio wavelengths. Much of what we know about its radiative behavior also relies on radio observations, but note that we know that its X-ray flux decreased by a factor of 50 between mid-2007 and early 2011, with an exponential timescale of $1 \mathrm{yr}$, following a presumed earlier outburst (Anderson et al. 2012). The high-cadence Parkes monitoring observations that we have presented here along with previously published radio results (Levin et al. 2010, 2012) allow us to consider the evolution of PSR J1622-4950 over many years and to place it in the context of other magnetars. 

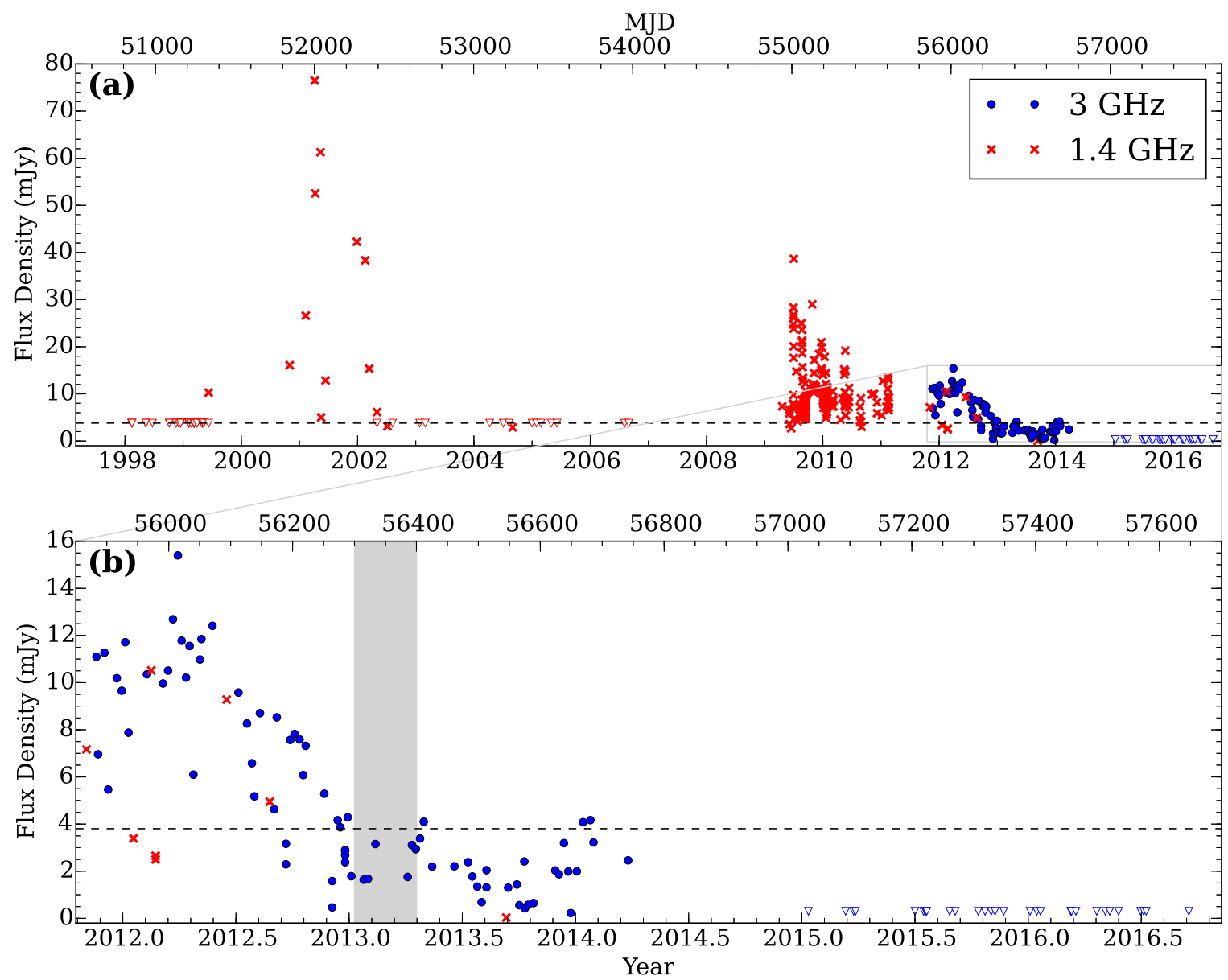

Figure 3. Top panel: flux density for each detection of PSR J1622-4950. Bottom panel: zoom-in on the flux density measurements from the new data presented in this work. Red crosses represent $1.4 \mathrm{GHz}$ observations and blue circles represent $3 \mathrm{GHz}$ observations. Open red triangles represent nondetections from the off-axis $1.4 \mathrm{GHz}$ prediscovery observations (Levin et al. 2010). They have a limiting flux density of $3.8 \mathrm{mJy}$ (see Section 3.2), indicated by the dashed line. Nondetections starting in early 2015 , largely at $3 \mathrm{GHz}$ with a limiting flux density of $0.3 \mathrm{mJy}$, are shown as downward-pointing blue triangles. Measurements between 2009 and mid2011 are those from Levin et al. (2012) multiplied by a scaling factor of 2.3 (see Section 3.2). The gray bar marks the period between MJD 56300 and MJD 56400 in order to illustrate the correlated behavior between the spin-down (Figure 4), flux density, and pulse profile evolution (Figure 2) at that time.

\subsection{Pulse Profile Variations}

The variability of the radio pulse profiles that we observed for PSR J1622-4950 between late 2011 and early 2014 (Section 3.1 and Figure 1) seems broadly comparable to that previously reported. However, Levin et al. (2010) presented rapidly changing pulse profiles from 2009 for which we had no counterparts (see their Figure 1) whereas the pulse profile variation that we observed was much smoother (Figures 1 and 2). It is possible that in this regard the magnetosphere of PSR J1622-4950 was more unsettled around the earlier time, which encompassed an epoch of rapidly decreasing X-ray flux (Anderson et al. 2012).

Correlated behavior between the profile variations and the spin-down state is observed in several young pulsars (e.g., Lyne et al. 2010; Keith et al. 2013). For these pulsars the spindown torque is correlated with changes in profile shape quantified by profile width (Lyne et al. 2010) or relative component heights (Keith et al. 2013). For PSR J1622-4950 we see a correlation that may be broadly similar: as the spindown torque decreases between late 2011 and early 2014, the width of the profile decreases as the second Gaussian component narrows and approaches the first component. We note however that this appears to be a continuous evolution, unlike correlated behavior between the discrete spin-down and profile states thus far discerned in some ordinary radio pulsars.
A similar secular decrease in the width of the pulse profile is not evident in other radio magnetars. Camilo et al. (2016) found that for XTE J1810-197 the profiles varied greatly right up to the disappearance of radio emission in late 2008 with no obvious secular evolution. Following its 2013 outburst, the magnetar SGR J1745-2900 showed a widening of its profile that stabilized after $\sim 100$ days (Lynch et al. 2015).

\subsection{Flux Density Evolution}

The flux density variability of PSR J1622-4950 is interesting, especially when compared to that of two other radio magnetars. Following its discovery in 2009, PSR J1622-4950 had a highly variable radio flux density (ranging over $\sim 3-40$ mJy at $1.4 \mathrm{GHz}$ ) that on average appeared to be on somewhat of a downward trend through early 2011 (Figure 3 and Levin et al. 2012). Our measurements until late 2012 (largely at $3 \mathrm{GHz}$ ) appear to be consistent with this description (Figure 3). Thereafter, however, a new regime took hold. Throughout 2013 and into early 2014, the measured flux density never exceeded $5 \mathrm{mJy}$ and in the latter half of 2013 was mostly below $2 \mathrm{mJy}$. Then sometime between 2014 March and 2015 January radio emission from PSR J1622-4950 ceased (or at least never rose above a flux density of $\approx 0.3 \mathrm{mJy}$ ). It remained in this state as of 2016 September. 
Table 1

Two Timing Solutions for PSR J1622-4950

\begin{tabular}{|c|c|c|}
\hline Parameter & Solution 1 & Solution 2 \\
\hline \multicolumn{3}{|c|}{ Timing Parameters } \\
\hline R. A. $(\mathrm{J} 2000)^{\mathrm{a}}$ & $16: 22: 44.8$ & $16: 22: 44.8$ \\
\hline Decl. $(\mathrm{J} 2000)^{\mathrm{a}}$ & $-49: 50: 54.4$ & $-49: 50: 54.4$ \\
\hline $\begin{array}{l}\text { Dispersion measure, DM } \\
\qquad\left(\mathrm{pc} \mathrm{cm}^{-3}\right)^{\mathrm{a}}\end{array}$ & 820 & 820 \\
\hline Spin frequency, $\nu\left(\mathrm{s}^{-1}\right)$ & $0.231115433(4)$ & $0.2311059204(8)$ \\
\hline Frequency derivative, $\dot{\nu}\left(\mathrm{s}^{-2}\right)$ & $-3.566(3) \times 10^{-13}$ & $-1.4827(7) \times 10^{-13}$ \\
\hline $\begin{array}{l}\text { Frequency second derivative, } \\
\quad \ddot{\nu}\left(\mathrm{s}^{-3}\right)\end{array}$ & $3.22(9) \times 10^{-21}$ & $1.09(3) \times 10^{-21}$ \\
\hline Epoch of frequency (MJD) & 56100.0 & 56563.0 \\
\hline Data span (MJD) & $55867-56334$ & $56385-56742$ \\
\hline Number of TOAs & 55 & 31 \\
\hline rms residual (phase) & 0.12 & 0.014 \\
\hline \multicolumn{3}{|c|}{ Derived Parameters } \\
\hline $\begin{array}{l}\text { Surface dipolar magnetic field, } \\
\quad B(\mathrm{G})\end{array}$ & $1.7 \times 10^{14}$ & $1.1 \times 10^{14}$ \\
\hline $\begin{array}{l}\text { Spin-down luminosity, } \dot{E} \\
\quad\left(\mathrm{erg} \mathrm{s}^{-1}\right)\end{array}$ & $3.2 \times 10^{33}$ & $1.4 \times 10^{33}$ \\
\hline Characteristic age, $\tau_{c}(\mathrm{kyr})$ & 10 & 25 \\
\hline
\end{tabular}

Note. Numbers in parentheses are TEMPO2 $1 \sigma$ uncertainties.

${ }^{a}$ Values fixed to those from Levin et al. (2010).

This behavior is reminiscent of that for the first radio magnetar, XTE J1810-197. Three years after its X-ray outburst and discovery (Ibrahim et al. 2004) the radio flux density was large, fluctuating greatly on a daily timescale and generally on a downward trend (the radio light curve for the three years following the X-ray outburst is essentially unknown; Camilo et al. 2007a). After one year at a low average flux density but still fluctuating greatly on daily timescales, the radio emission from XTE J1810-197 turned off in late 2008 and has not recurred (Camilo et al. 2016).

The flux density behavior of PSR J1622-4950 prior to its discovery in 2009, during the years 1998-2006, may have had a different character. It appears that the fluctuations may have been larger then, and more frequent, with no clearly discernible trends (see Figure 3, Section 3.2, and Figure 1 of Levin et al. 2010). Most of those early detections are from 2000 to 2002, with many nondetections in 1998-2000 and 2002-2006. However, the flux density limits in those years are above the flux densities for most of our detections in 2013 (Figure 3). We therefore have a range of possible interpretations spanning two extrema: (1) the radio pulsar did indeed turn on and off multiple times during 1998-2006, sometimes possibly on rapid timescales; (2) the radio pulsar was always on since at least 1998, although often in a faint state, below $3.8 \mathrm{mJy}$ (as during 2013), until it finally turned off in 2014.

In this regard it is instructive to consider the behavior of the second radio magnetar, 1E 1547.0-5408. When it was discovered in 2007, it was a bright and fluctuating radio source, consistently detectable, with X-ray flux decaying from a presumed prior outburst (Camilo et al. 2008). However, following two large X-ray outbursts in 2008 and 2009, radio pulses became detectable only sporadically (Camilo et al. 2009; Burgay et al. 2009) with months-long periods of no detection interspersed with sometimes hugely bright emission-and all the while the X-ray flux was decaying very slowly (F. Camilo et al. 2017, in preparation).

The current radio state of PSR J1622-4950 and its reduced flux density for one year prior to turning off (Figure 3 ) more closely resemble the behavior of XTE J1810-197 than that so far displayed by $1 \mathrm{E} 1547.0-5408$. This is particularly the case when considering the parallel behavior of the spin-down torque.

\subsection{Spin-down Behavior}

The known spin-down history of PSR J1622-4950 is summarized in Figure 5, where we reproduce the measurements of Levin et al. (2012) for 2009-2011 and add our own for 2011-2014 (see Figure 4(a) and Section 3.3). The detections in 1999-2003 (Figure 3(a)) are too sparse to yield $\dot{\nu}$ measurements.

We identify two regimes: for 2 yr until mid-2011, the torque on the neutron star (proportional to $|\dot{\nu}|$ ) varied erratically, both increasing and decreasing, and at very large rates; for the $2.4 \mathrm{yr}$ until early 2014, the torque decreased monotonically and generally at a lower rate than before. Overall, from 2009 until 2014 , the torque decreased by a factor of 8 .

While the torque that we measured during 2011-2014 decreased monotonically, it did not do so at a constant rate (Figure 4(a)). Until 2013 March, $|\dot{\nu}|$ decreased at the rate $\ddot{\nu}=3 \times 10^{-21} \mathrm{~s}^{-3}$. Then within approximately one month the torque decreased by almost a factor of 2 . Following this, its rate of change dropped markedly to $\ddot{\nu}=1 \times 10^{-21} \mathrm{~s}^{-3}$ (Table 1). Interestingly, early 2013 is also when the flux density plateaued at a low level (Figure 3(b) and Section 4.2). In the last year of radio emission $|\dot{\nu}|$ decreased by $20 \%$, a rate nearly one order of magnitude below the average for 2009-2014.

Such torque behavior is not unprecedented in magnetars. Following X-ray outbursts in 2002, 2007, and 2012, 1E 1048.1 -5937 displayed episodes where its $\dot{\nu}$ both increased and decreased repeatedly by up to a factor of 10 within $\sim 100-600$ days following the outbursts (Archibald et al. 2015). The torque variations then abated and the torque decreased to a relatively steady quiescent value. After both its 2008 and 2009 outbursts, 1E 1547.0-5408 experienced a rapid increase in spin-down torque (Dib et al. 2012), and large fluctuations continue (F. Camilo et al. 2017, in preparation). This behavior appears broadly comparable to that displayed by PSR J1622 -4950 until mid-2011 (Figure 5).

The history of the transient magnetar XTE J1810-197 since its one known X-ray outburst, detected in 2003, provides a more complete parallel to the overall behavior exhibited by PSR J1622-4950 since 2009. Following its outburst, XTE J1810-197 at first displayed erratic variations in torque (Gotthelf \& Halpern 2007). By 2006-2007, radio observations showed a large but monotonic decrease in $|\dot{\nu}|$ (Camilo et al. 2007a). The torque and radio flux density then (relatively) stabilized at low values for approximately one year, after which the detectable radio emission ceased (Camilo et al. 2016). On the whole, this seems to track what we have observed in PSR J1622-4950 since 2009 (Figures 5 and 3(b)).

\subsection{Twisted Magnetosphere Model}

In the twisted magnetosphere model for magnetar outbursts, the X-ray and radio emission are both caused by twists in the magnetosphere that can result from the shearing of the crust 


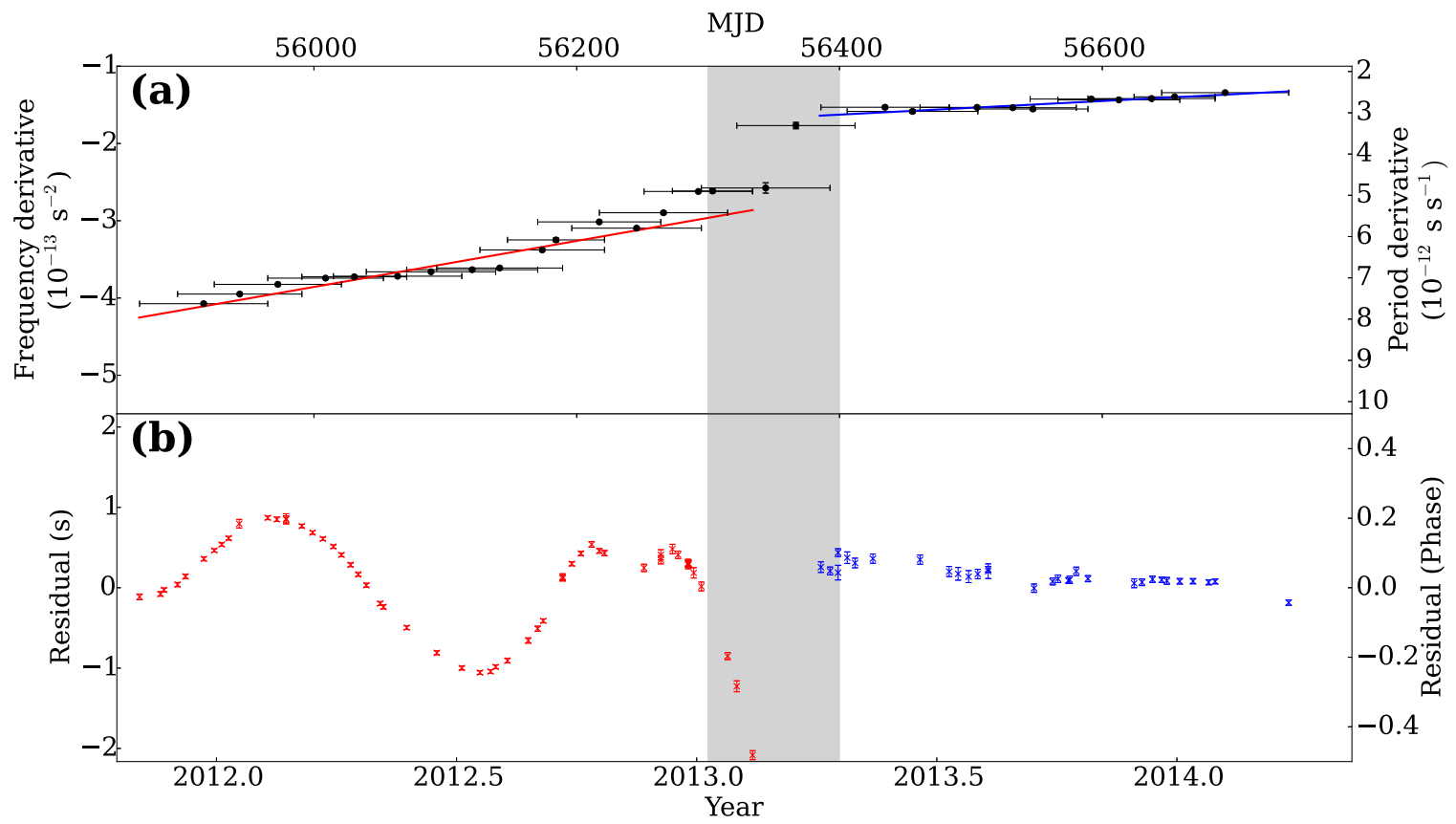

Figure 4. The rotation of PSR J1622-4950. Red and blue lines and points correspond to two simple $(\nu, \dot{\nu}, \ddot{\nu})$ timing solutions. (a) Frequency derivative. Black points represent short-term measurements of the frequency derivative (see Section 3.3). (b) Timing residuals for the two timing solutions (Table 1). The gray bar marks the period between MJD 56300 and MJD 56400 in order to illustrate the correlated behavior between the spin-down, flux density (Figure 3), and pulse profile evolution (Figure 2) at that time.

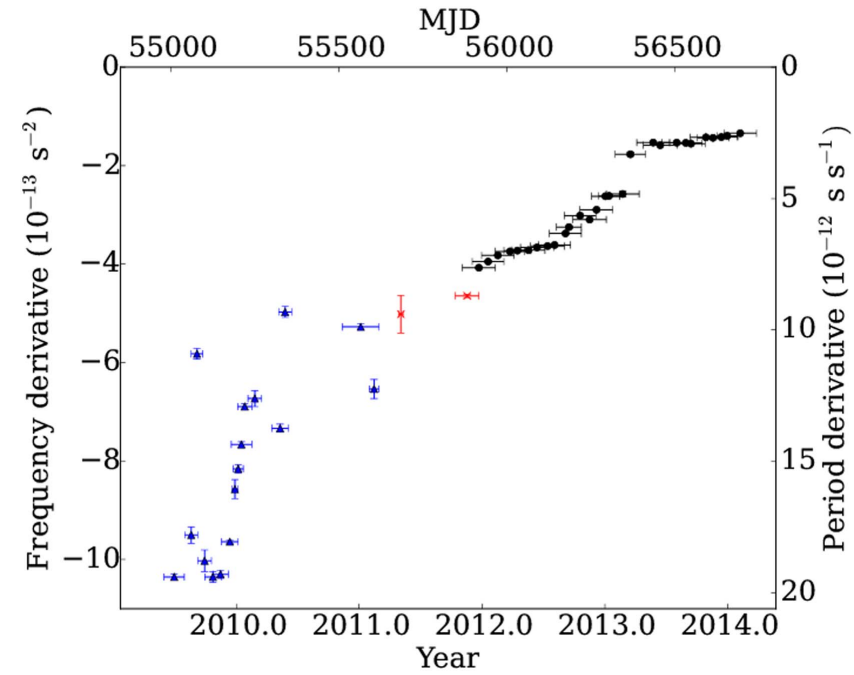

Figure 5. The frequency derivative of PSR J1622-4950 from discovery until radio disappearance. Measurements through early 2011, shown as blue triangles, are reproduced from Levin et al. (2012). Red crosses show frequency-derivative measurements derived from additional archival observations (Section 3.3); the second measurement was obtained using a combination of archival data and our initial 2011 observations. Frequency-derivative measurements from our 2011-2014 campaign (Figure 4(a)) are represented by black circles.

due to a starquake (Beloborodov 2009). The bundle of these twisted, closed field lines is called a "j-bundle." The X-rays result from a hotspot on the crust heated by currents driven by the $\mathrm{j}$-bundle, and the radio emission originates from the currents in the j-bundle itself.

Beloborodov (2009) applied his model to the outburst of XTE J1810-197, an event to which the behavior of PSR J1622-4950 displays some parallels. The nonmonotonic behavior in the spin-down torque observed for XTE J1810 -197 was attributed by Beloborodov (2009) to the increase of the twist angle at early times after the outburst. Depending on the initial conditions of the twist, the twist angle can grow as the $\mathrm{j}$-bundle is shrinking, causing the poloidal field lines to inflate, opening them at the light cylinder. This increase of the magnetic field at the light cylinder causes an increase in the spin-down torque. Once the twist angle reaches a maximum, the torque then decreases monotonically. This picture broadly fits the observed spin-down of PSR J1622-4950 (Figure 5), where an epoch of fluctuating torque after discovery was followed by a smooth monotonic decrease in $|\dot{\nu}|$ during 2011-2014.

In this model, we may expect the width of the radio pulse profile to decrease as the j-bundle shrinks. For PSR J1622 -4950 we do observe a decrease in the width of the trailing pulse profile component prior to the disappearance of detectable radio emission (Figure 2). However, the leading profile component remained constant in width, and a shrinking j-bundle therefore does not appear to entirely account for the observed evolution of the pulse profile.

\section{Conclusions}

We have presented $5 \mathrm{yr}$ of new Parkes radio observations of the magnetar PSR J1622-4950. We find that the torque on the neutron star decreased monotonically from late 2011 through 2014 March, decreasing at the smallest rate ever observed for this object starting in early 2013. The flux density, while variable, reached a relatively steady low level starting in early 2013 as well. Along with these decreases in flux density and torque, the pulse profile evolved in a secular fashion where the pulse became narrower as the secondary component approached the leading component. Sometime in the last 9 months of 2014, radio emission ceased and remained undetectable as of late 2016. This 
broadly parallels the behavior of the first radio magnetar, the transient XTE J1810-197.

The huge and rapid torque variations displayed by PSR J1622-4950 during 2009-2011 (Levin et al. 2012, and Figure 5), akin to those shown by other magnetars within a couple of years of X-ray outbursts (e.g., Archibald et al. 2015), together with its exponentially decaying X-ray flux during 2007-2011 (Anderson et al. 2012), argue for an undetected outburst occurring not long before mid-2007. However, the radio behavior of PSR J1622-4950 during 1999-2004 (Levin et al. 2010, and Figure 3) suggests an unsettled magnetosphere as far back as at least 1999 . We may therefore suppose that PSR J1622-4950 was not in quiescence for many years preceding its "2007" outburst.

PSR J1622-4950 is quite likely currently in as quiescent a state as it has been since at least 1999. This is supported by the smoothly decreasing torque in 2013-2014 and turnoff of any detectable radio emission by 2015 . We continue to monitor for the reactivation of radio pulsations with the Parkes telescope, although by analogy with other radio-detected magnetars this may not happen until a new X-ray outburst. The current X-ray state of PSR J1622-4950 is unknown; a measurement of its $\mathrm{X}$-ray flux would be very useful both to compare it to other magnetars and to provide a baseline for the next outburst that will surely arise.

The Parkes Observatory is part of the Australia Telescope, which is funded by the Commonwealth of Australia for operation as a National Facility managed by CSIRO. P. S. was supported by an Alexander Graham Bell Canada Graduate Scholarship from NSERC and a Schulich Graduate Fellowship from McGill University. P. S. holds a Covington Fellowship at DRAO.

\section{References}

Anderson, G. E., Gaensler, B. M., Slane, P. O., et al. 2012, ApJ, 751, 53

Archibald, R. F., Kaspi, V. M., Ng, C.-Y., et al. 2013, Natur, 497, 591

Archibald, R. F., Kaspi, V. M., Ng, C.-Y., et al. 2015, ApJ, 800, 33

Beloborodov, A. M. 2009, ApJ, 703, 1044

Burgay, M., Israel, G. L., Possenti, A., et al. 2009, ATel, 1913, 1

Camilo, F., Cognard, I., Ransom, S. M., et al. 2007a, ApJ, 663, 497

Camilo, F., Halpern, J. P., \& Ransom, S. M. 2009, ATel, 1907, 1

Camilo, F., Ransom, S., Halpern, J., et al. 2006, Natur, 442, 892

Camilo, F., Ransom, S. M., Halpern, J. P., et al. 2016, ApJ, 820, 110

Camilo, F., Ransom, S. M., Halpern, J. P., \& Reynolds, J. 2007b, ApJL, 666, L93

Camilo, F., Ransom, S. M., Peñalver, J., et al. 2007c, ApJ, 669, 561

Camilo, F., Reynolds, J., Johnston, S., Halpern, J. P., \& Ransom, S. M. 2008, ApJ, 679, 681

Dewey, R. J., Taylor, J. H., Weisberg, J. M., \& Stokes, G. H. 1985, ApJL, 294, L25

Dib, R., \& Kaspi, V. M. 2014, ApJ, 784, 37

Dib, R., Kaspi, V. M., Scholz, P., \& Gavriil, F. P. 2012, ApJ, 748, 3

Eatough, R. P., Falcke, H., Karuppusamy, R., et al. 2013, Natur, 501, 391

Gotthelf, E. V., \& Halpern, J. P. 2007, ApJL, 664, L35

Hobbs, G. M., Edwards, R. T., \& Manchester, R. N. 2006, MNRAS, 369, 655

Hotan, A. W., van Straten, W., \& Manchester, R. N. 2004, PASA, 21, 302

Ibrahim, A. I., Markwardt, C. B., Swank, J. H., et al. 2004, ApJL, 609, L21

Keith, M. J., Shannon, R. M., \& Johnston, S. 2013, MNRAS, 432, 3080

Levin, L., Bailes, M., Bates, S., et al. 2010, ApJL, 721, L33

Levin, L., Bailes, M., Bates, S. D., et al. 2012, MNRAS, 422, 2489

Lynch, R. S., Archibald, R. F., Kaspi, V. M., \& Scholz, P. 2015, ApJ, 806, 266

Lyne, A., Hobbs, G., Kramer, M., Stairs, I., \& Stappers, B. 2010, Sci, 329, 408

Manchester, R. N., Lyne, A. G., Camilo, F., et al. 2001, MNRAS, 328, 17

Mereghetti, S. 2008, A\&ARv, 15, 225

Olausen, S. A., \& Kaspi, V. M. 2014, ApJS, 212, 6

Ransom, S. M., Eikenberry, S. S., \& Middleditch, J. 2002, AJ, 124, 1788

Shannon, R. M., \& Johnston, S. 2013, MNRAS, 435, L29

Staveley-Smith, L., Wilson, W. E., Bird, T. S., et al. 1996, PASA, 13, 243

Woods, P. M., \& Thompson, C. 2006, in Compact Stellar X-ray Sources ed. W. H. G. Lewin \& M. van der Klis (Cambridge: Cambridge Univ. Press), 547 